\title{
Quantum control via enhanced shortcuts to adiabaticity
}

\author{
C. Whitty $\odot,{ }^{*}$ A. Kiely $\odot$, and A. Ruschhaupt $\odot$ \\ Department of Physics, University College Cork, Cork, Ireland
}

(Received 10 January 2020; accepted 14 April 2020; published 18 June 2020)

\begin{abstract}
Fast and robust quantum control protocols are often based on an idealized approximate description of the relevant quantum system. While this may provide a performance that is close to optimal, improvements can be made by incorporating elements of the full system representation. We propose a technique for such scenarios, called enhanced shortcuts to adiabaticity (eSTA). The eSTA method works for previously intractable Hamiltonians by providing an analytical correction to existing STA protocols. This correction can be easily calculated, and the resulting protocols are outside the class of STA schemes. We demonstrate the effectiveness of the method for three distinct cases: manipulation of an internal atomic state beyond the rotating-wave approximation, transport of a neutral atom in an optical Gaussian trap, and transport of two trapped ions in an anharmonic trap.
\end{abstract}

DOI: 10.1103/PhysRevResearch.2.023360

\section{INTRODUCTION}

The development of quantum technologies for a wide variety of applications is a rapidly growing field [1]. However, a critical roadblock in exploiting any quantum advantage is the fast and robust control of fragile quantum states. The process must be fast to avoid long interaction times with the external environment (decoherence) and stable to avoid accumulation of errors. These problems have been addressed by a number of distinct techniques, such as adiabatic methods [2], composite pulses [3-5], numerical optimal control [6-9], and shortcuts to adiabaticity $[10,11]$.

Shortcuts to adiabaticity (STA) are analytical methods to design the time dependence of the Hamiltonian to ensure effective adiabatic state evolution in finite time. STA methods have the advantage of providing physical insight into the control process as well as constructing a whole class of protocols that achieve the desired result. In combination with perturbation theory, the optimal protocol in this class can be found that is most stable regarding a relevant type of noise or imperfection [12-14]. There has also been work to improve protocols in a nonperturbative manner $[15,16]$, using variational methods $[17,18]$ and in combination with numerical optimal control [19-22].

STA methods have been used to control a variety of Hamiltonians, such as harmonic-oscillator potentials [23-25] and two-level [12,14], three-level [26,27], and four-level [28] systems. They have been utilized experimentally for trapped ions [29], superconducting qubits [30,31], nitrogen-vacancy centers [32,33], ultracold atoms [34], and Bose-Einstein

\footnotetext{
*c.whitty@umail.ucc.ie

Published by the American Physical Society under the terms of the Creative Commons Attribution 4.0 International license. Further distribution of this work must maintain attribution to the author(s) and the published article's title, journal citation, and DOI.
}

condensates [35]. However there are still many Hamiltonians that are not tractable with standard STA techniques. Our procedure is intended to deal with such cases.

In this paper, we provide an analytical enhancement to STA protocols inspired by techniques from numerical optimal control, termed enhanced shortcuts to adiabaticity (eSTA). There are several key benefits. First, the eSTA protocol provides higher fidelities than the STA protocol and may even be outside the original class of STA protocols. This represents a significant improvement over previous methods based on optimization inside the STA class [12]. Second, the resulting protocol is still completely analytical in nature, requiring no significant numerical computation or iterative procedure, and therefore the resulting protocols can provide further physical insight. In addition, eSTA protocols can also serve as good initial seeds for further numerical optimization (similar to recent attempts at utilizing human intuition [36]). As STA methods have been applied beyond quantum systems [11], for example in optical waveguides [37-39], classical mechanical systems [40-42], and statistical physics [43], eSTA has a broad range of applicability and in principle can be applied beyond the scope of quantum control.

In Sec. II we outline the details of the eSTA method, and the key result is summarized in Eq. (24). The following sections demonstrate the versatility of eSTA by applying it in three different settings that are all ubiquitous in quantum technologies. In Sec. III we consider population transfer in a two-level system beyond the rotating-wave approximation [44-47]. In Sec. IV we apply eSTA to the transport of a single neutral atom in an optical trap [48-50], and in Sec. V we apply eSTA to the transport of two trapped ions in an anharmonic trap [51-55].

\section{FORMALISM OF ENHANCED SHORTCUTS TO ADIABATICITY}

Consider a closed quantum system described by a Hamiltonian $\mathcal{H}_{\mu}$, which we will refer to as the system Hamiltonian. 

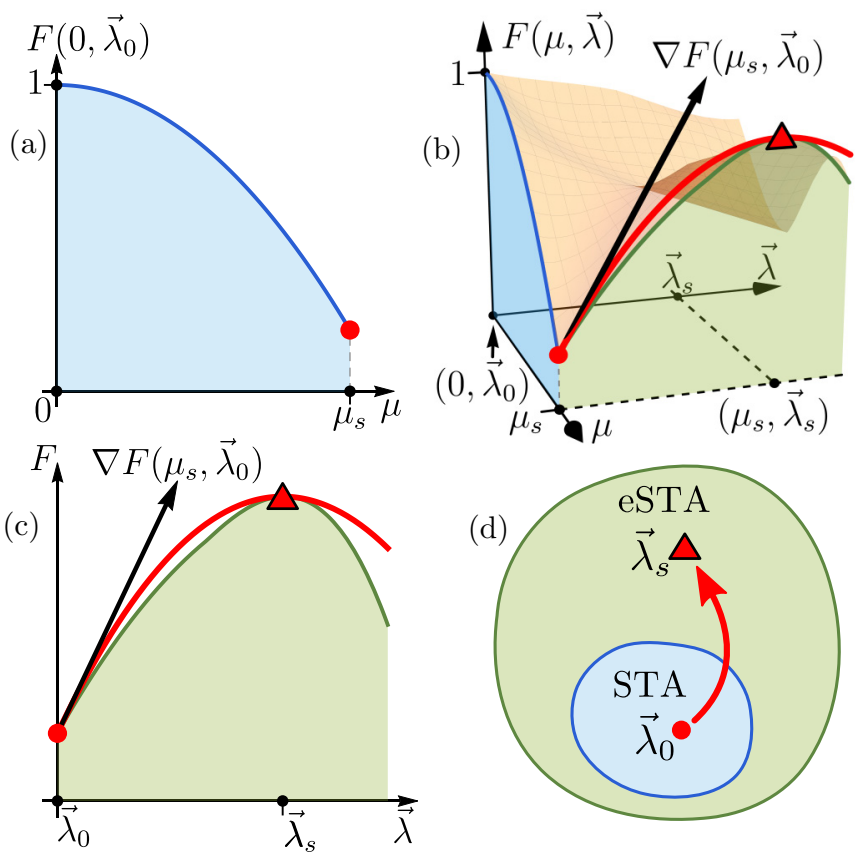

FIG. 1. Schematic overview of eSTA: (a) Fidelity using the STA protocol (defined by $\vec{\lambda}_{0}$ ) as a function of the Hamiltonian parametrization $\mu$. The red dot indicates the fidelity at the point $\mu_{s}$, which corresponds to the system Hamiltonian $\mathcal{H}_{\mu_{s}}$ using $\lambda_{0}$, where $\lambda_{0}$ is the STA protocol associated with the idealized Hamiltonian $\mathcal{H}_{0}$. (b) Surface diagram of the fidelity for different Hamiltonians $\mu$ and different control protocols $\vec{\lambda}$. The black arrow shows the gradient at $\left(\mu_{s}, \vec{\lambda}_{0}\right)$. The solid red line indicates the parabolic approximation with the red triangle located at its maximum. (c) Cross section of part (b), showing the fidelity for the system Hamiltonian as a function of the control parametrization $\lambda$. (d) Illustration that the set of control protocols eSTA (green, outer region) is larger than the set of STA methods (blue, inner region).

We assume that $\mathcal{H}_{\mu}$ has no known analytical quantum control procedure. Our goal is to change $\mathcal{H}_{\mu}$ in time so that the system evolves from the initial state $\left|\Psi_{0}\right\rangle$ at $t=0$ to the target state $\left|\Psi_{T}\right\rangle$ in a given total time $t_{f}$. The Schrödinger equation is $i \hbar \frac{\partial}{\partial t}|\Psi(t)\rangle=\mathcal{H}_{\mu}(\vec{\lambda} ; t)|\Psi(t)\rangle$, where the value of $\mu$ fixes the form of the Hamiltonian, and the time-dependent control of the system parameters is characterized by $\vec{\lambda}$. The fidelity for this evolution is $\mathrm{F}(\mu, \vec{\lambda})=\left|\left\langle\Psi_{T}\left|U_{\mu, \vec{\lambda}}\left(t_{f}, 0\right)\right| \Psi_{0}\right\rangle\right|^{2}$. First the system Hamiltonian $\mathcal{H}_{\mu}$ is approximated by an idealized, simpler Hamiltonian $\mathcal{H}_{0}$ where an STA method can be applied. The manipulations required for the STA protocol are parametrized by $\vec{\lambda}_{0} \in \mathbb{R}^{N}$. Our goal is to find $\vec{\lambda}$ such that the fidelity of this chosen evolution under $\mathcal{H}_{\mu}$ is improved, where the method of improvement is motivated by the GRAPE algorithm [6,56]. Clearly, just using the STA protocol that was designed for the idealized Hamiltonian $\mathcal{H}_{0}$ does not give perfect fidelity for the system Hamiltonian $\mathcal{H}_{\mu}$ [see the red dot in Fig. 1(a)]. However, we assume that the difference between the system and idealized Hamiltonians $\mu_{s}$ is small. Hence, we also assume that using the STA protocol $\vec{\lambda}_{0}$ for the system Hamiltonian is close to optimal; see Fig. 1(b).

To calculate how much and in what manner to alter the original STA scheme $\vec{\lambda}_{0}$, we calculate the gradient with respect to $\vec{\lambda}$ and assume that the fidelity behaves quadratically in the neighborhood of $\left(\mu_{s}, \vec{\lambda}_{0}\right)$; see Fig. 1(c). We also assume that the fidelity at the maximum of the parabola [see the red triangle in Figs. 1(b) and 1(c)] is approximately 1, i.e., $F\left(\mu_{s}, \vec{\lambda}_{0}+\vec{\epsilon}\right) \approx 1$. Hence this quadratic approximation gives

$$
F\left(\mu_{s}, \vec{\lambda}_{0}+\alpha \frac{\nabla F\left(\mu_{s}, \vec{\lambda}_{0}\right)}{\left|\nabla F\left(\mu_{s}, \vec{\lambda}_{0}\right)\right|}\right) \approx 1-c(\alpha-\epsilon)^{2},
$$

where $\epsilon=|\vec{\epsilon}|, c>0$, and $\alpha$ is a scalar parameter. We assume $\nabla F\left(\mu_{s}, \vec{\lambda}_{0}\right)$ and $F\left(\mu_{s}, \vec{\lambda}_{0}\right)$ are given such that we obtain $\epsilon$ from Eq. (1). Then we get a new control function given by $\vec{\lambda}_{s}=\vec{\lambda}_{0}+\vec{\epsilon}$, where the correction $\vec{\epsilon}$ is

$$
\vec{\epsilon} \approx \epsilon \frac{\nabla F\left(\mu_{s}, \vec{\lambda}_{0}\right)}{\left|\nabla F\left(\mu_{s}, \vec{\lambda}_{0}\right)\right|}=\frac{2\left[1-\mathrm{F}\left(\mu_{s}, \vec{\lambda}_{0}\right)\right]}{\left|\nabla F\left(\mu_{s}, \vec{\lambda}_{0}\right)\right|} \frac{\nabla F\left(\mu_{s}, \vec{\lambda}_{0}\right)}{\left|\nabla F\left(\mu_{s}, \vec{\lambda}_{0}\right)\right|} .
$$

Hence $\vec{\epsilon}$ describes the direction and distance from $\vec{\lambda}_{0}$ that should improve the fidelity.

\section{A. Calculation of eSTA correction term $\vec{\epsilon}$}

To calculate $\vec{\epsilon}$ we must estimate the gradient and the value of the fidelity at $\left(\mu_{s}, \vec{\lambda}_{0}\right)$ (see the red dot in Fig. 1). To derive these estimates, for simplicity we assume that the initial state $\left|\Psi_{0}\right\rangle$ and the final target state $\left|\Psi_{T}\right\rangle$ are independent of the parametrization $\mu$. Since STA methods can be applied for the idealized Hamiltonian, the solutions $\left|\chi_{n}(t)\right\rangle$ are known (using Lewis-Riesenfeld invariants for example). Since $U_{0, \vec{\lambda}_{0}}\left(t_{2}, t_{1}\right)$ is the time evolution for $\mu=0$, we have that $\left|\chi_{n}(t)\right\rangle=U_{0, \vec{\lambda}_{0}}(t, 0)\left|\chi_{n}(0)\right\rangle$ and $U_{0, \vec{\lambda}_{0}}(t, s)=$ $\sum_{n}\left|\chi_{n}(t)\right\rangle\left\langle\chi_{n}(s)\right|$. We assume $\left|\chi_{0}(0)\right\rangle=\left|\Psi_{0}\right\rangle$ and therefore $\left|\chi_{0}\left(t_{f}\right)\right\rangle=\left|\Psi_{T}\right\rangle$.

We now estimate the terms needed to calculate the correction, assuming that we can neglect higher-order contributions in both $\mu$ and $\vec{\epsilon}$. We start with a series expansion

$$
\mathcal{H}_{\mu}(\vec{\lambda} ; t)=\sum_{n=0}^{\infty} \mu^{n} \mathcal{H}^{(n)}(\vec{\lambda} ; t),
$$

where $\mathcal{H}^{(0)}(\vec{\lambda} ; t)=\mathcal{H}_{0}(\vec{\lambda} ; t)$. Time-dependent perturbation theory [57] provides a series expansion of the corresponding time-evolution operator,

$$
U_{\mu, \vec{\lambda}}\left(t_{2}, t_{1}\right)=\sum_{n=0}^{\infty} \mu^{n} U_{\vec{\lambda}}^{(n)}\left(t_{2}, t_{1}\right),
$$

where the exact form of the first, second, and third order can be found in Appendix.

\section{B. Approximation of $F\left(\mu, \vec{\lambda}_{0}\right)$}

We consider a series expansion of the fidelity in $\mu$,

$$
F\left(\mu, \vec{\lambda}_{0}\right)=\sum_{n=0}^{\infty} \mu^{n} F^{(n)} .
$$

By defining

$$
u_{j}=\left\langle\chi_{0}\left(t_{f}\right)\left|U_{\vec{\lambda}_{0}}^{(j)}\left(t_{f}, 0\right)\right| \chi_{0}(0)\right\rangle,
$$


we can express the coefficients in Eq. (5) generally as

$$
\begin{aligned}
F^{(n)} & =\sum_{k=0}^{n} u_{n-k} u_{k}^{*} \\
& = \begin{cases}\left|u_{n / 2}\right|^{2}+2 \operatorname{Re}\left(\sum_{k=0}^{\frac{n}{2}-1} u_{n-k} u_{k}^{*}\right), & n \text { is even, } \\
2 \operatorname{Re}\left(\sum_{k=0}^{(n-1) / 2} u_{n-k} u_{k}^{*}\right), & n \text { is odd. }\end{cases}
\end{aligned}
$$

Since the STA scheme works perfectly for the idealized Hamiltonian by construction, we have that $u_{0}=1$ and hence $F^{(0)}=\left|u_{0}\right|^{2}=1$. For the higher orders, one obtains (see Appendix for details)

$$
\begin{gathered}
F^{(1)}=0, \\
F^{(2)}=-\frac{1}{\hbar^{2}} \sum_{n=1}^{\infty}\left|\int_{0}^{t_{f}} d t \alpha_{n, 0}^{(1)}(t)\right|^{2}, \\
F^{(3)} \approx-\frac{2}{\hbar^{2}} \sum_{n=1}^{\infty} \operatorname{Re}\left[\left(\int_{0}^{t_{f}} d t \alpha_{n, 0}^{(1)}(t)\right)^{*}\left(\int_{0}^{t_{f}} d t \alpha_{n, 0}^{(2)}(t)\right)\right],
\end{gathered}
$$

where we have defined

$$
\alpha_{n, m}^{(j)}(t)=\left\langle\chi_{n}(t)\left|\mathcal{H}^{(j)}\left(\vec{\lambda}_{0} ; t\right)\right| \chi_{m}(t)\right\rangle .
$$

Using these results, the fidelity $F\left(\mu_{s}, \vec{\lambda}_{0}\right)$ can be approximated up to second order in $\mu_{s}$ as

$$
\begin{aligned}
F\left(\mu_{s}, \vec{\lambda}_{0}\right) & \approx 1-\frac{1}{\hbar^{2}} \sum_{n=1}^{\infty}\left|\int_{0}^{t_{f}} d t\left(\mu_{s} \alpha_{n, 0}^{(1)}(t)+\mu_{s}^{2} \alpha_{n, 0}^{(2)}(t)\right)\right|^{2} \\
& \approx 1-\frac{1}{\hbar^{2}} \sum_{n=1}^{\infty}\left|G_{n}\right|^{2}
\end{aligned}
$$

where $\mathcal{H}_{S}=\mathcal{H}_{\mu_{s}}$ and

$$
G_{n}=\int_{0}^{t_{f}} d t\left\langle\chi_{n}(t)\left|\left[\mathcal{H}_{S}\left(\vec{\lambda}_{0} ; t\right)-\mathcal{H}^{(0)}\left(\vec{\lambda}_{0} ; t\right)\right]\right| \chi_{0}(t)\right\rangle .
$$

\section{Approximation of $\nabla F\left(\mu, \vec{\lambda}_{0}\right)$}

The gradient of the fidelity with respect to $\vec{\lambda}$ is given by

$$
\begin{aligned}
\nabla F\left(\mu, \vec{\lambda}_{0}\right)= & 2 \operatorname{Re}\left[\left\langle\chi_{0}\left(t_{f}\right)\left|\nabla U_{\mu, \vec{\lambda}_{0}}\left(t_{f}, 0\right)\right| \chi_{0}(0)\right\rangle\right. \\
& \left.\times\left\langle\chi_{0}\left(t_{f}\right)\left|U_{\mu, \vec{\lambda}_{0}}\left(t_{f}, 0\right)\right| \chi_{0}(0)\right\rangle^{*}\right],
\end{aligned}
$$

and it can be expanded in $\mu$ as

$$
\nabla F\left(\mu, \lambda_{0}\right)=\sum_{n=0}^{\infty} \mu^{n} \overrightarrow{\mathcal{F}}^{(n)} .
$$

If we define

$$
\vec{v}_{j}=\left\langle\chi_{0}\left(t_{f}\right)\left|\nabla U_{\vec{\lambda}_{0}}^{(j)}\left(t_{f}, 0\right)\right| \chi_{0}(0)\right\rangle,
$$

then we can express the coefficients in Eq. (15) as

$$
\overrightarrow{\mathcal{F}}^{(n)}=2 \operatorname{Re}\left(\sum_{k=0}^{n} \vec{v}_{n-k} u_{k}^{*}\right) .
$$

Using time-dependent perturbation theory (similar to the previous subsection) and that

$$
\mathcal{H}^{(0)}(\vec{\lambda}) \approx \mathcal{H}^{(0)}\left(\vec{\lambda}_{0}\right)+\left(\vec{\lambda}-\vec{\lambda}_{0}\right) \cdot \nabla \mathcal{H}^{(0)}\left(\vec{\lambda}_{0}\right),
$$

we obtain simplified expressions for $\overrightarrow{\mathcal{F}}^{(0)}, \overrightarrow{\mathcal{F}}^{(1)}, \overrightarrow{\mathcal{F}}^{(2)}$, and $\overrightarrow{\mathcal{F}}^{(3)}$. Note that $\vec{v}_{0}$ is purely imaginary, hence it follows that $\overrightarrow{\mathcal{F}}^{(0)}=$ $\overrightarrow{0}$. The relevant following orders are detailed in Appendix, with

$$
\overrightarrow{\mathcal{F}}^{(1)}=-\frac{2}{\hbar^{2}} \sum_{n=1}^{\infty} \operatorname{Re}\left[\int_{0}^{t_{f}} d t \alpha_{n, 0}^{(1)}(t) \int_{0}^{t_{f}} d s \vec{\beta}_{n, 0}^{(0)}(s)^{*}\right]
$$

and

$$
\begin{aligned}
\overrightarrow{\mathcal{F}}^{(2)} \approx & -\frac{2}{\hbar^{2}} \sum_{n=1}^{\infty} \operatorname{Re}\left[\left(\int_{0}^{t_{f}} d t \vec{\beta}_{n, 0}^{(1)}(t)\right)^{*}\left(\int_{0}^{t_{f}} d t \alpha_{n, 0}^{(1)}(t)\right)\right. \\
& \left.+\left(\int_{0}^{t_{f}} d t \vec{\beta}_{n, 0}^{(0)}(t)\right)^{*}\left(\int_{0}^{t_{f}} d t \alpha_{n, 0}^{(2)}(t)\right)\right],
\end{aligned}
$$

where we have defined

$$
\vec{\beta}_{n, m}^{(j)}(t)=\left\langle\chi_{n}(t)\left|\nabla \mathcal{H}^{(j)}\left(\vec{\lambda}_{0} ; t\right)\right| \chi_{m}(t)\right\rangle .
$$

From these results, we get up to second order in $\mu_{s}$ that

$$
\begin{aligned}
& \nabla F\left(\mu_{s}, \lambda_{0}\right) \\
& \approx-\frac{2 \mu_{s}}{\hbar^{2}} \sum_{n=1}^{\infty} \operatorname{Re}\left\{\int_{0}^{t_{f}} d t\left[\mu_{s} \alpha_{n, 0}^{(1)}(t)+\mu_{s}^{2} \alpha_{n, 0}^{(2)}(t)\right]\right. \\
& \left.\quad \times \int_{0}^{t_{f}} d s\left[\vec{\beta}_{n, 0}^{(0)}(s)+\mu \vec{\beta}_{n, 0}^{(1)}(s)\right]^{*}\right\} \\
& \approx-\frac{2}{\hbar^{2}} \sum_{n=1}^{\infty} \operatorname{Re}\left(G_{n} \vec{K}_{n}^{*}\right),
\end{aligned}
$$

with

$$
\vec{K}_{n}=\int_{0}^{t_{f}} d t\left\langle\chi_{n}(t)\left|\nabla \mathcal{H}_{S}\left(\vec{\lambda}_{0} ; t\right)\right| \chi_{0}(t)\right\rangle .
$$

\section{D. eSTA control protocol}

From Eq. (2), we arrive at the key result of the paper, namely the analytical expression for the eSTA protocol,

$$
\vec{\lambda}_{s} \approx \vec{\lambda}_{0}-\frac{\left(\sum_{n=1}^{N}\left|G_{n}\right|^{2}\right)\left[\sum_{n=1}^{N} \operatorname{Re}\left(G_{n}^{*} \vec{K}_{n}\right)\right]}{\left|\sum_{n=1}^{N} \operatorname{Re}\left(G_{n}^{*} \vec{K}_{n}\right)\right|^{2}},
$$

where $G_{n}$ is given by Eq. (13), $K_{n}$ is given by Eq. (23), and we have truncated the infinite sums to the first $N$ terms. This truncation is shown to be valid in later practical examples.

We underline that $G_{n}$ and $\vec{K}_{n}$ can both be easily calculated as only the Hamiltonians and the known solutions for the idealized Hamiltonian $\mathcal{H}_{0}$ are involved. Note that the eSTA method provides protocols that can be outside the class of STA schemes [see Fig. 1(d)] and so it represents a significant improvement over previous perturbation-based optimization [12]. In the examples that follow, we demonstrate numerically that the derived eSTA protocols do indeed lie outside the class of STA solutions (see the inset of Fig. 2 and the left inset of Fig. 4). 


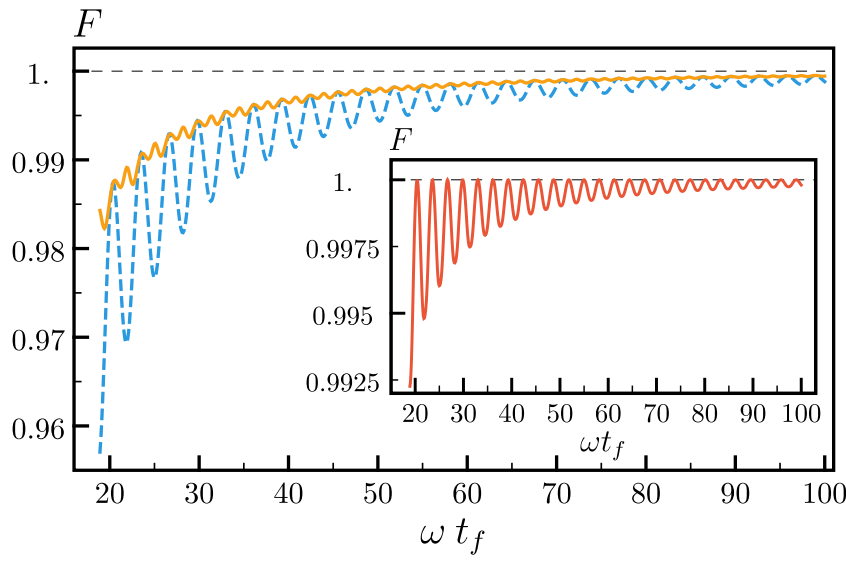

FIG. 2. Population inversion without the rotating-wave approximation: Fidelity $F$ vs final time $t_{f}$ for two-level Hamiltonian Eq. (25); the STA scheme $\vec{\lambda}_{0}$ (blue, dashed line) and the eSTA scheme $\vec{\lambda}_{s}$ (orange, solid line). Inset: Fidelity $F$ for the idealized Hamiltonian $\mathcal{H}_{0}$ using the eSTA scheme $\vec{\lambda}_{s}$ (red, solid line).

\section{POPULATION INVERSION WITHOUT THE ROTATING-WAVE APPROXIMATION}

As a first example, we consider the following system Hamiltonian:

$$
\mathcal{H}_{S}=\frac{\hbar}{2}\left(\begin{array}{cc}
-\delta(\vec{\lambda} ; t) & \Omega^{*}(\vec{\lambda} ; t)\left(1+e^{-2 i \omega t}\right) \\
\Omega(\vec{\lambda} ; t)\left(1+e^{2 i \omega t}\right) & \delta(\vec{\lambda} ; t)
\end{array}\right),
$$

which generically appears in many areas of quantum technologies. A common setting is that of two atomic states that are coupled by a classical light source (e.g., a laser) where the Rabi frequency $\Omega$ depends on the light amplitude, and the detuning $\delta$ depends on the light frequency $\omega$.

The terms $e^{ \pm 2 i \omega t}$ are typically neglected, which is known as the rotating-wave approximation (RWA) [58]. Our idealized Hamiltonian $\mathcal{H}_{0}$ is then just $\mathcal{H}_{S}$, where the terms $e^{ \pm 2 i \omega t}$ are set to zero.

While this approximation may work well for adiabatic methods, it will fail for fast nonadiabatic operations. Our goal is to use the eSTA method to provide fast population inversion even in the regime where the RWA does not hold (i.e., small values of $t_{f}$ ). This has been attempted previously using numerical methods [47]; however, here it will be done analytically.

Our initial scheme $\vec{\lambda}_{0}$ was derived to be stable concerning systematic errors in the Rabi frequency [12] (e.g., arising from the Gaussian profile of the laser), and it is given by

$$
\begin{aligned}
& \Omega\left(\vec{\lambda}_{0} ; t\right)=\frac{\pi}{t_{f}} \sqrt{1+16 \sin \left(\frac{\pi t}{t_{f}}\right)^{6}}, \\
& \delta\left(\vec{\lambda}_{0} ; t\right)=-8 \frac{\pi}{t_{f}} \sin \left(\frac{\pi t}{t_{f}}\right) \sin \left(\frac{2 \pi t}{t_{f}}\right) \frac{1+4 \sin \left(\frac{\pi t}{t_{f}}\right)^{6}}{1+16 \sin \left(\frac{\pi t}{t_{f}}\right)^{6}} .
\end{aligned}
$$

By design, this scheme gives perfect population inversion for the idealized Hamiltonian $\mathcal{H}_{0}$.

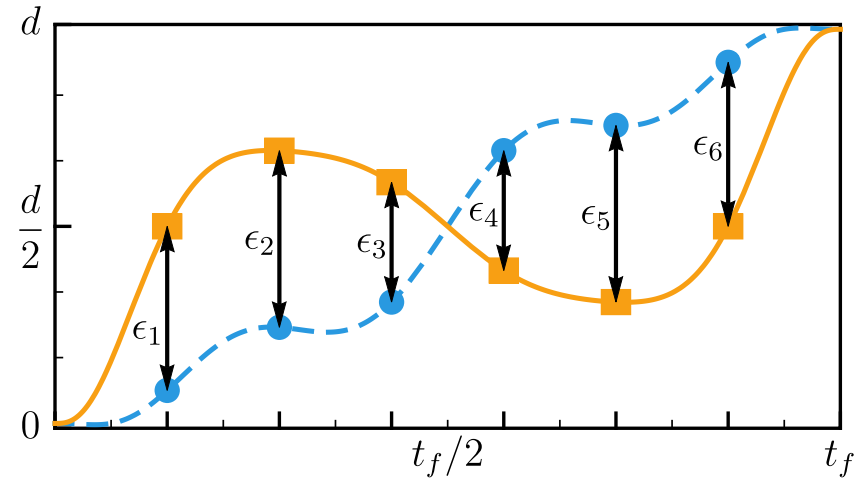

FIG. 3. Schematic diagram of a STA trap trajectory using $\vec{\lambda}_{0}$ (dashed blue line), and the improved eSTA trajectory using $\vec{\lambda}_{s}=$ $\vec{\lambda}_{0}+\vec{\epsilon}$ (solid orange line). The components of $\vec{\epsilon}$ are shown, where $\vec{\epsilon}=\left(\epsilon_{1}, \ldots, \epsilon_{6}\right)$. The transport time and distance are $t_{f}$ and $d$, respectively.

The scheme is modified as

$$
\begin{aligned}
\Omega(\vec{\lambda} ; t) & =\Omega\left(\vec{\lambda}_{0} ; t\right)+f_{1}(\vec{\epsilon} ; t), \\
\delta(\vec{\lambda} ; t) & =\delta\left(\vec{\lambda}_{0} ; t\right)+f_{2}(\vec{\epsilon} ; t),
\end{aligned}
$$

where $f_{1}$ and $f_{2}$ are the minimal polynomial functions, which fulfill $f_{i}\left(\vec{\epsilon} ; t^{\prime}\right)=0$ for $t^{\prime}=0, t_{f}$ and $f_{i}\left(\vec{\epsilon} ; \frac{j t_{f}}{5}\right)=$ $\epsilon_{4(i-1)+j}$, where we have chosen to use eight components $\vec{\epsilon}=\left(\epsilon_{n}\right)_{n=1, \ldots, 8}$. Note that $f_{1}(\overrightarrow{0}, t)=0$ and $f_{2}(\overrightarrow{0}, t)=0$. Since there are only two solutions in this setting, we can calculate $\vec{\epsilon}$ exactly, without any truncation. These solutions can be found analytically using Lewis-Riesenfeld invariants [12].

The fidelity using the STA and eSTA schemes for the system Hamiltonian $\mathcal{H}_{S}$ is shown in Fig. 2. For the shown final times $t_{f}$, the eSTA scheme outperforms the original STA scheme, since it always results in a higher or equal fidelity. The eSTA schemes are outside the set of STA control functions [see Fig. 1(d)]. This can been seen by calculating the fidelity of the eSTA schemes for the idealized Hamiltonian $\mathcal{H}_{0}$ (see the inset of Fig. 2). Since applying the STA scheme to the idealized Hamiltonian gives unit fidelity for all total times $t_{f}$ by construction, every fidelity value below 1 shows that the eSTA scheme is outside the set of STA schemes.

\section{SINGLE-PARTICLE TRANSPORT}

We consider transport of a particle in a one-dimensional trap over a distance $d$ in a total time $t_{f}$. The trap trajectory $q_{0}(\vec{\lambda}, t)$ is parametrized by a real valued control vector $\vec{\lambda}=$ $\left(\lambda_{1}, \ldots, \lambda_{6}\right)$, so that $q_{0}(0)=0, q_{0}\left(t_{f}\right)=d$, and $q_{0}(j / 7)=\lambda_{j}$ for $j=1, \ldots, 6$. A schematic example of a STA trajectory $q_{0}\left(\vec{\lambda}_{0}, t\right)$ and an eSTA trajectory $q_{0}\left(\vec{\lambda}_{s}, t\right)$ are shown in Fig. 3.

The system/idealized Hamiltonians are

$$
\mathcal{H}_{S / 0}=\frac{p^{2}}{2 m}+V_{S / 0}\left[x-q_{0}(\vec{\lambda}, t)\right]
$$




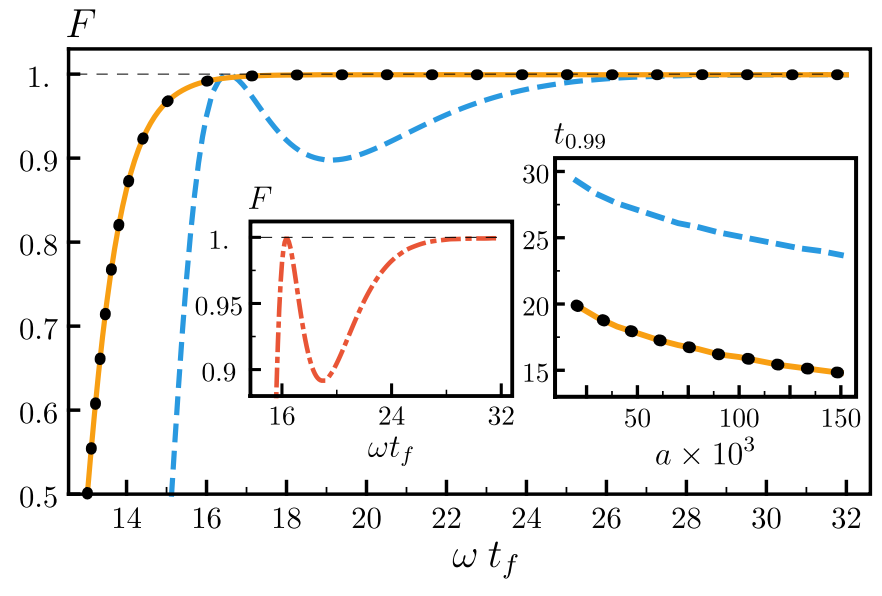

FIG. 4. Transport of a single particle: Using $a=100 \times 10^{3}$, the fidelity $F$ vs total time $t_{f}$ is shown for the STA scheme $\vec{\lambda}_{0}$ (blue, dashed line) and the eSTA scheme $\vec{\lambda}_{s}$, with $N=1$ (solid orange line) and $N=2$ (black dots). Left inset: Fidelity $F$ for the idealized Hamiltonian $\mathcal{H}_{0}$ using the eSTA scheme $\vec{\lambda}_{s}$ (red, dashed-dotted line). Right inset: threshold time $t_{0.99}$ vs $a$. Transport distance $d=1562 \sigma$.

where

$$
\begin{aligned}
& V_{S}(x)=U_{0}\left[1-\exp \left(-\frac{m \omega^{2}}{2 U_{0}} x^{2}\right)\right], \\
& V_{0}(x)=\frac{1}{2} m \omega^{2} x^{2},
\end{aligned}
$$

since $V(x) \rightarrow V_{0}(x)$ for $\mu=1 / a \rightarrow 0$, and $a=U_{0} /(\hbar \omega)$. There are known STA techniques for $\mathcal{H}_{0}$ to design trajectories $q_{0}(t)$ that give perfect fidelity, e.g., using Lewis-Riesenfeld invariants [24]. A known dynamical invariant for harmonic trap transport has the form

$$
I(t)=\frac{1}{2 m}\left(p-m \dot{q}_{c}\right)^{2}+\frac{1}{2} m \omega^{2}\left[x-m q_{c}(t)\right]^{2},
$$

where $q_{c}(t)$ must satisfy the auxiliary equation

$$
\ddot{q}_{c}+\omega^{2}\left(q_{c}-q_{0}\right)=0 .
$$

This equation relates the physical trap trajectory $q_{0}(t)$ with the particle's classical path $q_{c}(t)$ (which parametrizes the state). $q_{0}(t)$ can be inverse-engineered using boundary conditions and an appropriately chosen $q_{c}(t)$ via Eq. (32). To ensure the system is in the ground state after transport and that the trap is stationary, we require the boundary conditions $q_{c}(0)=$ $0, q_{c}\left(t_{f}\right)=0, \frac{d^{n} q_{c}\left(t^{\prime}\right)}{d t^{n}}=0$ for $n=1, \ldots, 4$ at $t^{\prime}=0, t_{f}$. We set $q_{c}(t)=\sum_{n=0}^{10} c_{n} t^{n}$, and we find $q_{0}(t)$ from Eq. (32). To implement eSTA, we need only to calculate $\vec{\epsilon}$ using $G_{n}$ and $\vec{K}_{n}$. The eigenstates $\left|\chi_{n}(s)\right\rangle$ are known analytically from [24], and so the integrals $G_{n}$ and $K_{n}$ can be calculated for each $n$. In the following, we will show that using $N=1$ is sufficient.

In Fig. 4, the fidelity $F$ is shown versus different final times $t_{f}$ using the STA transport scheme $\vec{\lambda}_{0}$ (blue, dashed line) and the eSTA transport scheme $\vec{\lambda}_{s}$ (using $N=1$, orange, solid line). This was calculated numerically where the time evolution was performed using the Fourier split-operator method and the initial ground state was found by imaginary-time evolution. For generality, we use natural units: the frequency $\omega$ of the approximated harmonic potential as the inverse time unit, $\sigma=\sqrt{\hbar /(m \omega)}$ as the length scale, and $\hbar \omega$ as the energy scale. We set the transport distance to $d=1562 \sigma$ and $a=$ $100 \times 10^{3}$. The chosen dimensionless values can correspond to different physical settings, for example to $\mathrm{a}{ }^{87} \mathrm{Rb}$ atom within an optical Gaussian trap of $U_{0}=0.4 \mathrm{mK}$, a trap width of $w=2 \sqrt{a} \sigma=334 \mu \mathrm{m}$ (purposely chosen very wide to be far from the regime of classical and adiabatic motion), and a transport distance of $d=825 \mu \mathrm{m}$.

We see a significant improvement in transport fidelity using eSTA in comparison with STA. For extremely short times, the approximation breaks down and neither STA nor eSTA produces good fidelity. For longer times, the system approaches adiabaticity and the two schemes converge. Clearly it is sufficient to consider just the first order, as the results for $N=1$ and 2 are identical; see Fig. 4 . While not a requirement, we note that the symmetry of the STA trajectory is preserved by the eSTA protocol.

To highlight the fact that these eSTA schemes are outside the set of STA schemes [Fig. 1(d)], we calculate the fidelity using $\lambda_{s}$ for the idealized Hamiltonian $\mathcal{H}_{0}$ (left inset in Fig. 4, red dashed-dotted line). By design, every STA scheme must give a fidelity of exactly 1 , which is not necessarily true when using the eSTA protocol.

To examine the dependence on $a$, we look at a threshold time $t_{0.99}$ that is defined as the time such that the fidelity $F \geqslant 0.99$ for all final times $t_{f} \geqslant t_{0.99}$. The right inset plot shows this threshold time $t_{0.99}$ versus different values of $a$. We see that the eSTA threshold time (orange, solid line) decreases with increasing $a$ and is always much lower than the corresponding STA threshold time (blue, dashed line). We have also investigated other potentials that produced qualitatively similar results to the Gaussian trap. This underlines the broad applicability of eSTA for single-particle transport.

\section{TRANSPORT OF TWO IONS INCLUDING COULOMB INTERACTION}

We now consider transport of two interacting (Coulomb) ions with equal mass $m$ and charge $+e$ in a one-dimensional Gaussian trap $V_{S}$ (as in the previous case), over a distance $d$, and in total time $t_{f}$. The coordinates of the ions in the laboratory frame are given by $x_{1}$ and $x_{2}$. We assume that $x_{1}>x_{2}$, and we treat the ions as distinguishable. We define $M=2 m$ and move to center-of-mass and relative coordinates defined by $x_{\mathrm{c}}=\left(x_{1}+x_{2}\right) / 2$ and $x_{\mathrm{r}}=\left(x_{1}-x_{2}\right) / 2$. The system/idealized Hamiltonian then becomes

$$
\begin{aligned}
\mathcal{H}_{S / 0}= & \frac{p_{\mathrm{c}}^{2}}{2 M}+\frac{p_{\mathrm{r}}^{2}}{2 M}+\frac{C}{2 x_{\mathrm{r}}}+V_{S / 0}\left[x_{\mathrm{c}}+x_{\mathrm{r}}-q_{0}(\vec{\lambda}, t)\right] \\
& +V_{S / 0}\left[x_{\mathrm{c}}-x_{\mathrm{r}}-q_{0}(\vec{\lambda}, t)\right],
\end{aligned}
$$

where $C=e^{2} / 4 \pi \epsilon_{0}$ and $V_{0}(x)=\frac{1}{2} M \omega^{2} x^{2}$ in this case. As $\mathcal{H}_{0}$ becomes separable in center-of-mass and relative coordinates, STA techniques need only be applied to the center of mass [53]. For the eSTA scheme, we assume that the relative distance between the ions is constant and equal to the stationary equilibrium distance when calculating $G_{n}$ and $\vec{K}_{n}$.

In Fig. 5, the fidelity $F$ is shown versus different final times $t_{f}$ using the STA transport scheme $\vec{\lambda}_{0}$ (dashed, blue 


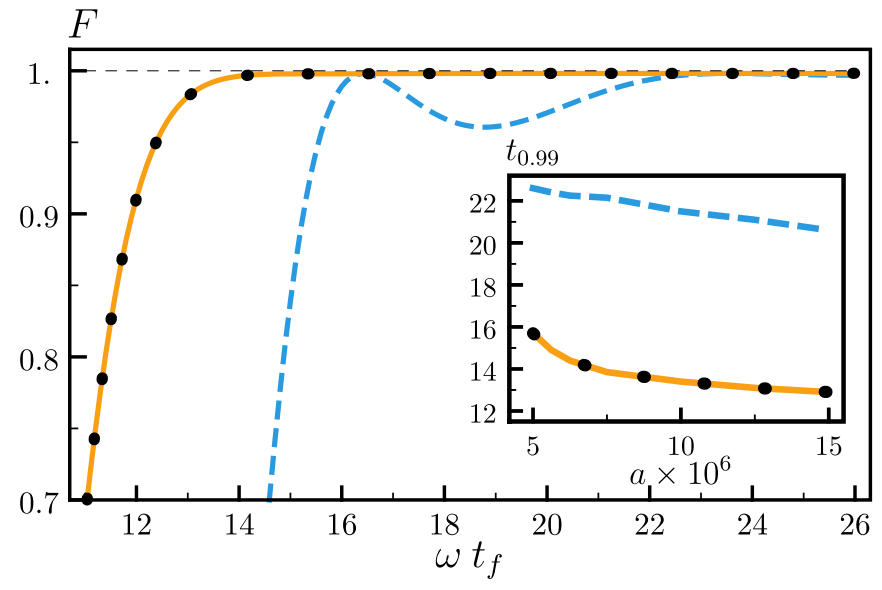

FIG. 5. Transport of two ions including Coulomb interaction: $a=10^{7}$ for the outer figure, $C=7.35 \times 10^{7}$; otherwise see caption of Fig. 4.

line) and the eSTA transport scheme $\vec{\lambda}_{s}$ (using $N=1$, orange, solid line). We set the transport distance to $d=1562 \sigma, a=$ $10^{7}$, and the dimensionless Coulomb constant $\tilde{C}=\frac{e^{2}}{4 \pi \epsilon_{0}} \frac{1}{\sigma \hbar \omega}=$ $7.35 \times 10^{7}$. These dimensionless values can correspond to different physical settings, for example transport of two ${ }^{9} \mathrm{Be}^{+}$ ions over $370 \mu \mathrm{m}$ in a surface ion trap of depth $U_{0}=0.8 \mathrm{meV}$ and frequency $0.13 \mathrm{MHz}$ (again a large trap width).

Similar to the previous case, we see a significant improvement in transport fidelity based on eSTA in comparison with
STA. For longer times, the system approaches adiabaticity and the two schemes converge. It is sufficient to consider just the first order (i.e., set $N=1$ when calculating the eSTA scheme); to check this, we also plot the result for $N=2$ (black dots), which gives identical results. As before, we find that the eSTA schemes are outside the set of STA schemes. The inset of Fig. 5, shows the threshold time $t_{0.99}$ versus $a$. We see that the eSTA threshold time (using $N=1$, orange, solid line) decreases with increasing $a$ and it is always significantly lower than the corresponding STA threshold time (blue dashed line).

\section{CONCLUSION}

In this paper we have presented an analytic extension to previous STA quantum control methods. We have demonstrated through three complementary examples relevant to quantum technologies that this method can be applied to improve performance and achieve physical insight. Further work could focus on deriving strict criteria and uncertainty relations for when the method works effectively. The eSTA procedure could be extended in several ways, such as to condensates, open systems, and even beyond the scope of quantum control.

\section{ACKNOWLEDGMENTS}

We are grateful to D. Rea for useful discussion and for commenting on the manuscript. C.W. acknowledges support by the Irish Research Council (GOIPG/2017/1846).

\section{APPENDIX: ESTIMATIONS of $F\left(\mu, \vec{\lambda}_{0}\right)$ and $\nabla F\left(\mu, \vec{\lambda}_{0}\right)$}

In this Appendix, we provide further details concerning the estimations of $F\left(\mu, \vec{\lambda}_{0}\right)$ and $\nabla F\left(\mu, \vec{\lambda}_{0}\right)$, which are used to derive the main formula of enhanced shortcuts to adiabaticity (eSTA). A series expansion of the time-evolution operator of the system Hamiltonian is

$$
U_{\mu, \vec{\lambda}}\left(t_{2}, t_{1}\right)=\sum_{n=0}^{\infty} \mu^{n} U_{\vec{\lambda}}^{(n)}\left(t_{2}, t_{1}\right)
$$

where the first order is

$$
U_{\vec{\lambda}}^{(1)}\left(t_{2}, t_{1}\right)=-\frac{i}{\hbar} \int_{t_{1}}^{t_{2}} d t U_{\vec{\lambda}}^{(0)}\left(t_{2}, t\right) \mathcal{H}^{(1)}(\vec{\lambda} ; t) U_{\vec{\lambda}}^{(0)}\left(t, t_{1}\right),
$$

the second order is

$$
\begin{aligned}
U_{\vec{\lambda}}^{(2)}\left(t_{2}, t_{1}\right)= & -\frac{1}{\hbar^{2}} \int_{t_{1}}^{t_{2}} d t \int_{t_{1}}^{t} d s U_{\vec{\lambda}}^{(0)}\left(t_{2}, t\right) \mathcal{H}^{(1)}(\vec{\lambda} ; t) U_{\vec{\lambda}}^{(0)}(t, s) \mathcal{H}^{(1)}(\vec{\lambda} ; s) U_{\vec{\lambda}}^{(0)}\left(s, t_{1}\right) \\
& -\frac{i}{\hbar} \int_{t_{1}}^{t_{2}} d t U_{\vec{\lambda}}^{(0)}\left(t_{2}, t\right) \mathcal{H}^{(2)}(\vec{\lambda} ; t) U_{\vec{\lambda}}^{(0)}\left(t, t_{1}\right),
\end{aligned}
$$

and finally the third order is

$$
\begin{aligned}
U_{\vec{\lambda}}^{(3)}\left(t_{2}, t_{1}\right) & =\frac{i}{\hbar^{3}} \int_{t_{1}}^{t_{2}} d t \int_{t_{1}}^{t} d s \int_{t_{1}}^{s} d u U_{\vec{\lambda}}^{(0)}\left(t_{2}, t\right) \mathcal{H}^{(1)}(\vec{\lambda} ; t) U_{\vec{\lambda}}^{(0)}(t, s) \mathcal{H}^{(1)}(\vec{\lambda} ; s) U_{\vec{\lambda}}^{(0)}(s, u) \mathcal{H}^{(1)}(\vec{\lambda} ; u) U_{\vec{\lambda}}^{(0)}\left(u, t_{1}\right) \\
& -\frac{1}{\hbar^{2}} \int_{t_{1}}^{t_{2}} d t \int_{t_{1}}^{t} d s U_{\vec{\lambda}}^{(0)}\left(t_{2}, t\right)\left[\mathcal{H}^{(1)}(\vec{\lambda} ; t) U_{\vec{\lambda}}^{(0)}(t, s) \mathcal{H}^{(2)}(\vec{\lambda} ; s)+\mathcal{H}^{(2)}(\vec{\lambda} ; t) U_{\vec{\lambda}}^{(0)}(t, s) \mathcal{H}^{(1)}(\vec{\lambda} ; s)\right] U_{\vec{\lambda}}^{(0)}\left(s, t_{1}\right) \\
& -\frac{i}{\hbar} \int_{t_{1}}^{t_{2}} d t U_{\vec{\lambda}}^{(0)}\left(t_{2}, t\right) \mathcal{H}^{(3)}(\vec{\lambda} ; t) U_{\vec{\lambda}}^{(0)}\left(t, t_{1}\right) .
\end{aligned}
$$


Note also that the matrix elements defined in Eqs. (11) and (21) obey the relations $\alpha_{n, m}^{(j)}(t)=\alpha_{m, n}^{(j)}(t)^{*}$ and $\vec{\beta}_{n, m}^{(j)}(t)=\vec{\beta}_{m, n}^{(j)}(t)^{*}$. Hence it follows that $\alpha_{n, n}^{(j)}(t)$ and $\vec{\beta}_{n, n}^{(j)}(t)$ are real.

\section{Approximation of $F\left(\mu, \vec{\lambda}_{0}\right)$}

From Eq. (A2), we get

$$
u_{1}=-\frac{i}{\hbar} \int_{0}^{t_{f}} d t\left\langle\chi_{0}(t)\left|\mathcal{H}^{(1)}\left(\vec{\lambda}_{0} ; t\right)\right| \chi_{0}(t)\right\rangle=-\frac{i}{\hbar} \int_{0}^{t_{f}} d t \alpha_{0,0}^{(1)}(t) .
$$

As $u_{1}$ is purely imaginary, it follows that $F^{(1)}=0$.

Now by using Eq. (A3), we get

$$
u_{2}=-\frac{1}{\hbar^{2}} \int_{0}^{t_{f}} d t \int_{0}^{t} d s\left\langle\chi_{0}(t)\left|\mathcal{H}^{(1)}(\vec{\lambda} ; t) U_{\vec{\lambda}_{0}}^{(0)}(t, s) \mathcal{H}^{(1)}(\vec{\lambda} ; s)\right| \chi_{0}(s)\right\rangle-\frac{i}{\hbar} \int_{0}^{t_{f}} d t\left\langle\chi_{n}(t)\left|\mathcal{H}^{(2)}\left(\vec{\lambda}_{0} ; t\right)\right| \chi_{0}(t)\right\rangle .
$$

Using $U_{\vec{\lambda}_{0}}^{(0)}(t, s)=\sum_{n}\left|\chi_{n}(t)\right\rangle\left\langle\chi_{n}(s)\right|$ simplifies this to

$$
u_{2}=-\frac{1}{\hbar^{2}} \int_{0}^{t_{f}} d t \int_{0}^{t} d s \sum_{n} \alpha_{0, n}^{(1)}(t) \alpha_{n, 0}^{(1)}(s)-\frac{i}{\hbar} \int_{0}^{t_{f}} d t \alpha_{0,0}^{(2)}(t) .
$$

After a suitable transformation of the integration variables, we can also write this as

$$
u_{2}=-\frac{1}{2 \hbar^{2}} \int_{0}^{t_{f}} d t \sum_{n}\left[\int_{0}^{t} d s \alpha_{0, n}^{(1)}(t) \alpha_{n, 0}^{(1)}(s)+\int_{t}^{t_{f}} d s \alpha_{0, n}^{(1)}(s) \alpha_{n, 0}^{(1)}(t)\right]-\frac{i}{\hbar} \int_{0}^{t_{f}} d t \alpha_{0,0}^{(2)}(t) .
$$

This form will be useful for calculating $F^{(2)}$ since

$2 \operatorname{Re}\left(u_{2}\right)=-\frac{1}{\hbar^{2}} \sum_{n} \int_{0}^{t_{f}} d t \int_{0}^{t_{f}} d s \operatorname{Re}\left[\alpha_{0, n}^{(1)}(t) \alpha_{n, 0}^{(1)}(s)\right]=-\frac{1}{\hbar^{2}} \sum_{n} \operatorname{Re}\left[\int_{0}^{t_{f}} d t \alpha_{0, n}^{(1)}(t) \int_{0}^{t_{f}} d s \alpha_{n, 0}^{(1)}(s)\right]=-\frac{1}{\hbar^{2}} \sum_{n}\left|\int_{0}^{t_{f}} d t \alpha_{n, 0}^{(1)}(t)\right|^{2}$

because $\alpha_{0, n}^{(1)}=\alpha_{n, 0}^{(1)^{*}}$ and $\alpha_{0,0}^{(2)}(t)$ is real. Finally, we get

$$
F^{(2)}=-\frac{1}{\hbar^{2}} \sum_{n=1}^{\infty}\left|\int_{0}^{t_{f}} d t \alpha_{n, 0}^{(1)}(t)\right|^{2}
$$

In a similar way by using Eq. (A4), we arrive at

$$
u_{3}=\frac{i}{\hbar^{3}} \int_{t_{1}}^{t_{f}} d t \int_{0}^{t} d s \int_{0}^{s} d u \sum_{n, m} \alpha_{0, n}^{(1)}(t) \alpha_{n, m}^{(1)}(s) \alpha_{n, 0}^{(1)}(u)-\frac{1}{\hbar^{2}} \int_{0}^{t_{f}} d t \int_{0}^{t} d s \sum_{n}\left[\alpha_{0, n}^{(1)}(t) \alpha_{n, 0}^{(2)}(s)+\alpha_{0, n}^{(2)}(t) \alpha_{n, 0}^{(1)}(s)\right]-\frac{i}{\hbar} \int_{0}^{t_{f}} d t \alpha_{0,0}^{(3)} \text {. }
$$

We use this together with previous results to calculate $F^{(3)}$. However, we restrict ourselves to the contributions that involve double integrals (while ignoring the contributions with three integrals over time). In this way,

$$
F^{(3)} \approx-\frac{2}{\hbar^{2}} \sum_{n=1}^{\infty} \operatorname{Re}\left[\left(\int_{0}^{t_{f}} d t \alpha_{n, 0}^{(1)}(t)\right)^{*}\left(\int_{0}^{t_{f}} d t \alpha_{n, 0}^{(2)}(t)\right)\right] .
$$

\section{Approximation of $\nabla F\left(\mu, \vec{\lambda}_{0}\right)$}

It will be useful to define a generalization of $\vec{v}_{0}$, namely

$$
\vec{W}_{n, m}\left(t_{2}, t_{1}\right) \equiv\left\langle\chi_{n}\left(t_{2}\right)\left|\nabla U_{\vec{\lambda}_{0}}^{(0)}\left(t_{2}, t_{1}\right)\right| \chi_{m}\left(t_{1}\right)\right\rangle .
$$

Using Eq. (21), we can simplify these terms to

$$
\vec{W}_{n, m}\left(t_{2}, t_{1}\right)=-\frac{i}{\hbar} \int_{t_{1}}^{t_{2}} d t \vec{\beta}_{n, m}^{(0)}(t)
$$

Note that $\vec{v}_{0}=\vec{W}_{0,0}\left(t_{f}, 0\right)$, and since $\vec{W}_{0,0}$ is purely imaginary, it follows again that $\overrightarrow{\mathcal{F}}^{(0)}=\overrightarrow{0}$.

From Eq. (A2), we get

$$
\vec{v}_{1}=-\frac{i}{\hbar} \int_{0}^{t_{f}} d t\left\langle\chi_{0}\left(t_{f}\right)\left|\nabla\left[U_{\vec{\lambda}}^{(0)}\left(t_{f}, t\right) \mathcal{H}^{(1)}(\vec{\lambda} ; t) U_{\vec{\lambda}}^{(0)}(t, 0)\right]\right|_{\vec{\lambda}=\vec{\lambda}_{0}} \mid \chi_{0}(0)\right\rangle .
$$


Using the product rule and inserting identities $\mathbb{1}=\sum_{n}\left|\chi_{n}(t)\right\rangle\left\langle\chi_{n}(t)\right|$, we arrive at

$$
\begin{aligned}
\vec{v}_{1} & =-\frac{i}{\hbar} \int_{0}^{t_{f}} d t\left\{\sum_{n}\left[\vec{W}_{0, n}\left(t_{f}, t\right) \alpha_{n, 0}^{(1)}(t)+\alpha_{0, n}^{(1)}(t) \vec{W}_{n, 0}(t, 0)\right]+\vec{\beta}_{0,0}^{(1)}(t)\right\} \\
& =-\frac{1}{\hbar^{2}} \sum_{n} \int_{0}^{t_{f}} d t\left[\int_{t}^{t_{f}} d s \vec{\beta}_{0, n}^{(0)}(s) \alpha_{n, 0}^{(1)}(t)+\int_{0}^{t} d s \alpha_{0, n}^{(1)}(t) \vec{\beta}_{n, 0}^{(0)}(s)\right]-\frac{i}{\hbar} \int_{0}^{t_{f}} d t \vec{\beta}_{0,0}^{(1)}(t) .
\end{aligned}
$$

To find $\overrightarrow{\mathcal{F}}^{(1)}$, we first calculate

$$
2 \operatorname{Re}\left(\vec{v}_{1}\right)=-\frac{2}{\hbar^{2}} \sum_{n} \operatorname{Re}\left[\int_{0}^{t_{f}} d t \alpha_{n, 0}^{(1)}(t) \int_{0}^{t_{f}} d s \vec{\beta}_{n, 0}^{(0)}(s)^{*}\right]
$$

and

Combining these two results gives

$$
2 \operatorname{Re}\left(\vec{v}_{0} u_{1}^{*}\right)=\frac{2}{\hbar^{2}} \operatorname{Re}\left[\int_{0}^{t_{f}} d t \alpha_{0,0}^{(1)}(t) \int_{0}^{t_{f}} d s \vec{\beta}_{0,0}^{(0)}(s)^{*}\right] .
$$

$$
\overrightarrow{\mathcal{F}}^{(1)}=-\frac{2}{\hbar^{2}} \sum_{n=1}^{\infty} \operatorname{Re}\left[\int_{0}^{t_{f}} d t \alpha_{n, 0}^{(1)}(t) \int_{0}^{t_{f}} d s \vec{\beta}_{n, 0}^{(0)}(s)^{*}\right] .
$$

Using Eq. (A3) and a similar calculation as above, we arrive at

$$
\begin{aligned}
\vec{v}_{2}= & \frac{i}{\hbar^{3}} \int_{0}^{t_{f}} d t \int_{0}^{t} d s \sum_{n, m}\left[\int_{t}^{t_{f}} d u \vec{\beta}_{0, n}^{(0)}(u) \alpha_{n, m}^{(1)}(t) \alpha_{m, 0}^{(1)}(s)+\int_{s}^{t} d u \alpha_{0, n}^{(1)}(t) \vec{\beta}_{n, m}^{(0)}(u) \alpha_{m, 0}^{(1)}(s)+\int_{0}^{s} d u \alpha_{0, n}^{(1)}(t) \alpha_{n, m}^{(1)}(s) \vec{\beta}_{m, 0}^{(0)}(u)\right] \\
& -\frac{1}{\hbar^{2}} \int_{0}^{t_{f}} d t \int_{0}^{t} d s\left\{\sum_{n}\left[\vec{\beta}_{0, n}^{(1)}(t) \alpha_{n, 0}^{(1)}(s)+\alpha_{0, n}^{(1)}(t) \vec{\beta}_{n, 0}^{(1)}(s)\right]\right\} \\
& -\frac{1}{\hbar^{2}} \sum_{n} \int_{0}^{t_{f}} d t\left[\int_{t}^{t_{f}} d s \vec{\beta}_{0, n}^{(0)}(s) \alpha_{n, 0}^{(2)}(t)+\int_{0}^{t} d s \alpha_{0, n}^{(2)}(t) \vec{\beta}_{n, 0}^{(0)}(s)\right]-\frac{i}{\hbar} \int_{0}^{t_{f}} d t \vec{\beta}_{0,0}^{(2)}(t) .
\end{aligned}
$$

Similar to the calculation of $\overrightarrow{\mathcal{F}}^{(2)}$, we neglect contributions involving three integrals over time, which results in

$$
\overrightarrow{\mathcal{F}}^{(2)} \approx-\frac{2}{\hbar^{2}} \sum_{n=1}^{\infty} \operatorname{Re}\left[\left(\int_{0}^{t_{f}} d t \vec{\beta}_{n, 0}^{(1)}(t)\right)^{*}\left(\int_{0}^{t_{f}} d t \alpha_{n, 0}^{(1)}(t)\right)+\left(\int_{0}^{t_{f}} d t \vec{\beta}_{n, 0}^{(0)}(t)\right)^{*}\left(\int_{0}^{t_{f}} d t \alpha_{n, 0}^{(2)}(t)\right)\right] .
$$

[1] W. P. Schleich et al., Appl. Phys. B 122, 130 (2016).

[2] N. V. Vitanov, A. A. Rangelov, B. W. Shore, and K. Bergmann, Rev. Mod. Phys. 89, 015006 (2017).

[3] B. T. Torosov, E. S. Kyoseva, and N. V. Vitanov, Phys. Rev. A 92, 033406 (2015).

[4] S. S. Ivanov and N. V. Vitanov, Phys. Rev. A 92, 022333 (2015).

[5] B. T. Torosov and N. V. Vitanov, Phys. Rev. A 90, 012341 (2014).

[6] N. Khaneja, T. Reiss, C. Kehlet, T. Schulte-Herbrüggen, and S. J. Glaser, J. Magn. Reson. 172, 296 (2005).

[7] S. J. Glaser, U. Boscain, T. Calarco, C. P. Koch, W. Köckenberger, R. Kosloff, I. Kuprov, B. Luy, S. Schirmer, T. Schulte-Herbrüggen, D. Sugny, and F. K. Wilhelm, Eur. Phys. J. D 69, 279 (2015).

[8] S. van Frank, M. Bonneau, J. Schmiedmayer, S. Hild, C. Gross, M. Cheneau, I. Bloch, T. Pichler, A. Negretti, T. Calarco, and S. Montangero, Sci. Rep. 6, 34187 (2016).

[9] P. Doria, T. Calarco, and S. Montangero, Phys. Rev. Lett. 106, 190501 (2011).
[10] E. Torrontegui, S. Ibáñez, S. Martínez-Garaot, M. Modugno, A. del Campo, D. Guéry-Odelin, A. Ruschhaupt, X. Chen, and J. G. Muga, Adv. At., Mol., Opt. 62, 117 (2013).

[11] D. Guéry-Odelin, A. Ruschhaupt, A. Kiely, E. Torrontegui, S. Martínez-Garaot, and J. G. Muga, Rev. Mod. Phys. 91, 045001 (2019).

[12] A. Ruschhaupt, X. Chen, D. Alonso, and J. G. Muga, New J. Phys. 14, 093040 (2012).

[13] A. Ruschhaupt and J. G. Muga, J. Mod. Opt. 61, 828 (2014).

[14] A. Kiely and A. Ruschhaupt, J. Phys. B 47, 115501 (2014).

[15] F. Impens and D. Guéry-Odelin, Sci. Rep. 9, 4048 (2019).

[16] A. Levy, A. Kiely, J. G. Muga, R. Kosloff, and E. Torrontegui, New J. Phys. 20, 025006 (2018).

[17] H. Saberi, T. Opatrný, K. Mølmer, and A. del Campo, Phys. Rev. A 90, 060301(R) (2014).

[18] D. Sels and A. Polkovnikov, Proc. Natl. Acad. Sci. USA 114, E3909 (2017). 
[19] R. Corgier, S. Amri, W. Herr, H. Ahlers, J. Rudolph, D. GuéryOdelin, E. M. Rasel, E. Charron, and N. Gaaloul, New J. Phys. 20, 055002 (2018).

[20] H. L. Mortensen, J. J. W. H. Sørensen, K. Mølmer, and J. F. Sherson, New J. Phys. 20, 025009 (2018).

[21] S. Campbell, G. De Chiara, M. Paternostro, G. M. Palma, and R. Fazio, Phys. Rev. Lett. 114, 177206 (2015).

[22] S. Martínez-Garaot, A. Ruschhaupt, J. Gillet, T. Busch, and J. G. Muga, Phys. Rev. A 92, 043406 (2015).

[23] X. Chen, A. Ruschhaupt, S. Schmidt, A. del Campo, D. GuéryOdelin, and J. G. Muga, Phys. Rev. Lett. 104, 063002 (2010).

[24] E. Torrontegui, S. Ibáñez, X. Chen, A. Ruschhaupt, D. GuéryOdelin, and J. G. Muga, Phys. Rev. A 83, 013415 (2011).

[25] A. Kiely, J. P. L. McGuinness, J. G. Muga, and A. Ruschhaupt, J. Phys. B 48, 075503 (2015).

[26] X. Chen and J. G. Muga, Phys. Rev. A 86, 033405 (2012).

[27] A. Benseny, A. Kiely, Y. Zhang, T. Busch, and A. Ruschhaupt, EPJ Quantum Technol. 4, 3 (2017).

[28] A. Kiely, A. Benseny, T. Busch, and A. Ruschhaupt, J. Phys. B 49, 215003 (2016).

[29] S. An, D. Lv, A. del Campo, and K. Kim, Nat. Commun. 7, 12999 (2016).

[30] T. Wang, Z. Zhang, L. Xiang, Z. Jia, P. Duan, W. Cai, Z. Gong, Z. Zong, M. Wu, J. Wu, L. Sun, Y. Yin, and G. Guo, New J. Phys. 20, 065003 (2018).

[31] T. Wang, Z. Zhang, L. Xiang, Z. Jia, P. Duan, Z. Zong, Z. Sun, Z. Dong, J. Wu, Y. Yin, and G. Guo, Phys. Rev. Appl. 11, 034030 (2019).

[32] J. Zhang, J. H. Shim, I. Niemeyer, T. Taniguchi, T. Teraji, H. Abe, S. Onoda, T. Yamamoto, T. Ohshima, J. Isoya, and D. Suter, Phys. Rev. Lett. 110, 240501 (2013).

[33] J. Kölbl, A. Barfuss, M. S. Kasperczyk, L. Thiel, A. A. Clerk, H. Ribeiro, and P. Maletinsky, Phys. Rev. Lett. 122, 090502 (2019).

[34] J.-F. Schaff, P. Capuzzi, G. Labeyrie, and P. Vignolo, New J. Phys. 13, 113017 (2011).

[35] J.-F. Schaff, X.-L. Song, P. Capuzzi, P. Vignolo, and G. Labeyrie, Europhys. Lett. 93, 23001 (2011).

[36] J. J. W. H. Sørensen, M. K. Pedersen, M. Munch, P. Haikka, J. H. Jensen, T. Planke, M. G. Andreasen, M. Gajdacz, K. Mølmer, A. Lieberoth, and J. F. Sherson, Nature (London) 532, 210 (2016).

[37] S. Longhi, Laser Photon. Rev. 3, 243 (2009).

[38] T.-Y. Lin, F.-C. Hsiao, Y.-W. Jhang, C. Hu, and S.-Y. Tseng, Opt. Express 20, 24085 (2012).
[39] H.-C. Chung, K.-S. Lee, and S.-Y. Tseng, Opt. Express 25, 13626 (2017).

[40] J. Deng, Q.-h. Wang, Z. Liu, P. Hänggi, and J. Gong, Phys. Rev. E 88, 062122 (2013).

[41] S. Deffner, C. Jarzynski, and A. del Campo, Phys. Rev. X 4, 021013 (2014).

[42] S. González-Resines, D. Guéry-Odelin, A. Tobalina, I. Lizuain, E. Torrontegui, and J. G. Muga, Phys. Rev. Appl. 8, 054008 (2017).

[43] D. Guéry-Odelin, J. G. Muga, M. J. Ruiz-Montero, and E. Trizac, Phys. Rev. Lett. 112, 180602 (2014).

[44] J. Scheuer, X. Kong, R. S. Said, J. Chen, A. Kurz, L. Marseglia, J. Du, P. R. Hemmer, S. Montangero, T. Calarco, B. Naydenov, and F. Jelezko, New J. Phys. 16, 093022 (2014).

[45] P. London, P. Balasubramanian, B. Naydenov, L. P. McGuinness, and F. Jelezko, Phys. Rev. A 90, 012302 (2014).

[46] G. D. Fuchs, V. V. Dobrovitski, D. M. Toyli, F. J. Heremans, and D. D. Awschalom, Science 326, 1520 (2009).

[47] S. Ibáñez, Y.-C. Li, X. Chen, and J. G. Muga, Phys. Rev. A 92 , 062136 (2015).

[48] S. Kuhr, W. Alt, D. Schrader, M. Müller, V. Gomer, and D. Meschede, Science 293, 278 (2001).

[49] J. Beugnon, C. Tuchendler, H. Marion, A. Gaëtan, Y. Miroshnychenko, Y. R. P. Sortais, A. M. Lance, M. P. A. Jones, G. Messin, A. Browaeys, and P. Grangier, Nat. Phys. 3, 696 (2007).

[50] C. Muldoon, L. Brandt, J. Dong, D. Stuart, E. Brainis, M. Himsworth, and A. Kuhn, New J. Phys. 14, 073051 (2012).

[51] A. Walther, F. Ziesel, T. Ruster, S. T. Dawkins, K. Ott, M. Hettrich, K. Singer, F. Schmidt-Kaler, and U. Poschinger, Phys. Rev. Lett. 109, 080501 (2012).

[52] R. Bowler, J. Gaebler, Y. Lin, T. R. Tan, D. Hanneke, J. D. Jost, J. P. Home, D. Leibfried, and D. J. Wineland, Phys. Rev. Lett. 109, 080502 (2012).

[53] M. Palmero, E. Torrontegui, D. Guéry-Odelin, and J. G. Muga, Phys. Rev. A 88, 053423 (2013).

[54] M. Palmero, R. Bowler, J. P. Gaebler, D. Leibfried, and J. G. Muga, Phys. Rev. A 90, 053408 (2014).

[55] X.-J. Lu, M. Palmero, A. Ruschhaupt, X. Chen, and J. G. Muga, Phys. Scr. 90, 074038 (2015).

[56] N. Wu, A. Nanduri, and H. Rabitz, Phys. Rev. B 91, 041115(R) (2015).

[57] A. Messiah, Quantum Mechanics (Dover, New York, 2014).

[58] L. Allen and J. H. Eberly, Optical Resonance and Two-Level Atoms (Dover, New York, 1987). 